\title{
Bladder Sparing Therapy for BCG Failures - I - Intravesical Immunotherapy
}

\author{
Edward M. Messing* \\ University of Rochester Medical Center, Rochester, NY, USA
}

Between 15 and $20 \%$ of patients with newly diagnosed urothelial cancer (UC) of the bladder will have high grade (HG) non-muscle invasive (NMI) disease including carcinoma-in-situ (CIS) and stage Ta and T1 tumors [1]. After transurethral resection of the cancer (TURBT), patients may undergo re-TURBT, but if no muscle invasive (MI) cancer is found most will receive an induction course of 6 weekly intravesical instillations of Bacillus Calmette Guerin (BCG). If at the first post BCG surveillance cystoscopy no cancer is found, they usually receive 3 weekly instillations of BCG every 6 months for 3 years [2]. If persistent or recurrent NMI cancer is found, they will usually receive another 6 week re-induction course of BCG without [3] or with alpha interferon [4].

Persistence or recurrence of any HG cancer at 6 months after the initial TURBT (termed "BCG unresponsive or resistant" disease) is generally an indication for cystectomy. However, bladder cancer is a disease of the elderly, and usually arises in patients with numerous comorbidities [5]. Thus, some UC patients are not candidates for cystectomy, and some patients who are medical "candidates" decline to undergo it. Treatment options for these patients are limited. Only Valrubicin has been approved by the US Food and Drug Administration (FDA) for salvage intravesical therapy for patients with CIS after BCG, even though $20 \%$ or fewer patients so treated remained recurrence-free by 12 months [6]. Studies of bladder preservation therapies for BCG unresponsive or "relapsing" (recurrence of HG NMI UC after

\footnotetext{
*Correspondence to: Edward M. Messing, University of Rochester Medical Center, Rochester, NY, USA. Tel.: +1 585275 3345; E-mail: Edward.messing@urmc.rochester.edu.
}

a tumor free interval of less than 12 months after the index TURBT) cancer [7] have reported that courses of intravesical instillations of a variety of single agents including chemotherapies and biological preparations effect $<25 \%$ two year recurrence free survival (RFS), and combination chemotherapies, usually gemcitabine with either mitomycin $\mathrm{C}$ or docetaxel provide $\leq 35 \%$ two year RFS [7]. Phase II studies with other novel agents, [8] radiation therapy with systemic chemotherapy, and systemic checkpoint inhibitors are also underway, but not completed.

Two recent articles report novel immunotherapies for BCG unresponsive and relapsing HG NMI UC $[9,10]$.

Shore and co-workers in the Society of Urologic Oncology Clinical Trials Consortium, building upon a phase $1 \mathrm{~b}$ trial reported from the MD Anderson Cancer Center, [11] randomized 40 patients with primarily BCG unresponsive HG NMI UC to receive $75 \mathrm{ml}$ of $10^{11}$ viral particles $(\mathrm{VP}) / \mathrm{ml}$ or $3 \times 10^{11}$ $\mathrm{vp} / \mathrm{ml}$ of rAd-INF $\alpha$-Syn 3 . Over $90 \%$ of participants had at least two prior induction courses of BCG.

rAd-INF $\alpha$ is a replication deficient recombinant adenovirus encoding the human INF $\alpha 2 b$ gene, which was administered with Syn3, a polyamide surfactant which enhances adenoviral transduction of the urothelium. rAd-INF $\alpha$-Syn 3 was instilled intravesically one time several weeks after the index TURBT, and one month after negative cystoscopy, cytology and biopsy (if clinically indicated) at months 3, 6 and 9 after the index TURBT. 33\% of patients receiving the lower dose and $36.8 \%$ receiving the higher dose of $\mathrm{rAd}-\mathrm{INF} \alpha$-Syn 3 were free of recurrence at 12 months. While these results may seem modest, it should be noted that over half the patients at each 
dose level had BCG unresponsive disease, indicating that they were at very high risk of recurrence. All patients had elevated urinary interferon levels 2 and 4 days after each instillation, but interferon level did not correlate with response. Over $70 \%$ of those who were recurrence-free at 12 months had positive antiadenoviral antibody titers ( $>4$ times pre-dose titers), while only $24 \%$ of those who experienced a recurrence did.

Urinary urgency, frequency, dysuria and fatigue occurred in $32.5-40 \%$ of patients, but almost $80 \%$ of adverse events (AE) were transient and primarily not severe. Grade 3 AEs occurred in $22 \%$ and most were not drug related. There were no grade 4 or 5 AEs.

Interferon $\alpha$ has anti-angiogenic and pro-apoptotic properties, and induces dendritic cell maturation promoting tumor cell recognition by $\mathrm{T}$ cells and NK cells; but how it helps prevent bladder tumor recurrence is not precisely known. Because interferon $\alpha$ induces programmed death ligand 1 (PD-L1) expression, it is possible that combining rAd-INF $\alpha$-Syn 3 with a systemic PD-L1 inhibitor would have additive or even synergistic efficacy.

Steinberg and colleagues described an intravesical combination of $1 / 3$ strength BCG, 50 million units of interferon alpha, 22 million units of interleukin-2 and $250 \mathrm{mcg}$ of subcutaneously injected granulocytemacrophage colony stimulating factor (quadruple immunotherapy = QIT) [10]. QIT was given weekly for 3 weeks, and then, after a two week hiatus, another 3 weekly treatments were administered. This was done primarily, for BCG relapsing disease. Complete responders underwent repeat 3 weekly QIT treatments at months 3,9 , and 15 using lower doses of BCG.

Forty-seven of 52 patients $(90 \%)$ had some side effect, mostly urinary frequency, urgency and dysuria, and fatigue. In $9(17.5 \%)$ a delay in treatment was required. Three patients $(6 \%)$ could not tolerate the full induction course. However $55 \%$ of patients were disease free at 12 months, and $53 \%$ at 24 months. Presence of CIS or age $>80$ years did not affect results.

Thirteen patients underwent cystectomy and 5 patients who recurred were deemed not to be cystectomy candidates. However, of the patients who underwent cystectomy, while 2 had pT3 disease, none had positive surgical margins or positive lymph nodes, implying there was a window of time after initial BCG relapse where patients could undergo bladder salvage therapy and still have curative surgery if needed.
In future Paper Alerts other bladder sparing approaches to BCG resistant and relapsing HG NMI UC will be discussed, as study results are published. However, the FDA recognizes the great need in this patient subgroup, and is trying to find mechanisms to rapidly approve agents for these patients.

\section{REFERENCES}

[1] Messing EM, Madeb R, Young T, et al. Long-term outcome of hematuria home screening for bladder cancer in men. Cancer 2006:107:2173-9.

[2] Lamm DL, et al. Maintenance bacillus-calmette-guerin immunotherapy for recurrent $\mathrm{Ta}, \mathrm{T} 1$ and carcinoma-in-situ transitional cell carcinoma of the bladder. A randomized Southwest Oncology Group study. J Urol 2000;163(4): 1124-9.

[3] Catalona WJ, Hudson MA, Gillen DP, et al. Risks and benefits of repeated courses of intravesical bacillus-calmetteguerin therapy for superficial bladder cancer. J Urol 1987; 137:220-4.

[4] Nepple KG, Lightfoot AJ, Rosevear HM, et al. Bladder Cancer Genitourinary Study G. Bacillus calmette guerin with or without interferon alpha- $2 \mathrm{~b}$ and megadose versus recommended daily allowance vitamins during induction and maintenance intravesical treatment of non-muscle invasive bladder cancer. J Urol 2010;184:1915-9.

[5] Scosyrev E, Wu G, Golijanin D, et al. Non-bladder cancer mortality in patients with urothelial cancer of the bladder. Urol Oncol 2013:13(5):656-63.

[6] Ignatoff JM, Chen YH, Greenberg RE, et al. Phase II study of intravesical therapy with AD32 in patients with papillary urothelial carcinoma or carcinoma in situ (CIS) refractory to prior therapy with bacillus calmette-guerin (E3897): A trial of the Eastern Cooperative Oncology Group. Urol Onc 2009;27:496-501.

[7] Steinberg RL, Thomas LJ, Nepple KG. Intravesical and alternative bladder preservation therapies in the management of non-muscle invasive bladder cancer unresponsive to bacillus calmette-guerin. Urologic Onc 2016;34:279-289.

[8] Huang J, Schisler J, Wong HC, et al. Intravesical ALT-803 for BCG-unresponsive bladder cancer - A Case Report. Urology Case Reports 2007;14:15-17.

[9] Shore ND, Boorjan SA, Canter DJ, et al. Intravesical rAd-If $\alpha /$ Syn 3 for patients with high grade, bacillus calmette-guerin refractory or relapsed non-muscle invasive bladder cancer. A phase II randomized study. J Clin Oncol. 2017:JCO2017723064. doi: 10.1200/JCO.2017.72.3064. [Epub ahead of print]

[10] Steinberg RL, Nepple KG, Valeur K, et al. Quadruple immunotherapy of bacillus calmette guerin, interferon, interleukin-2 and granulocyte-macrophage colony-stimulating factor as a salvage therapy for nonmuscle-invasive bladder cancer. Urologic Oncology 2017. pii: S1078-1439(17)30386-1. doi: 10.1016/j.urolonc.2017. 07.024. [Epub ahead of print]

[11] Navai N, Benedict WF, Zhang G, et al. Phase Ib trial to evaluate tissue response to a second dose of intravesical recombinant adenoviral interferon $\alpha 2 b$ formulated in Syn3 for failures of bacillus-calmette-guerin (BCG) therapy in non-muscle invasive bladder cancer. Ann Surg Oncol 2016;23:4110-14. 